\title{
Análisis del significado psicológico sobre el concepto ciencia en profesores de enseñanza básica y media: una aproximación desde una experiencia de formación continua
}

Por: Dr. Cristián Manuel Aguilar Correa ${ }^{1}$,

Universidad Católica del Maule, Chile, ORCID: 0000-0002-7231-0628

Recibido: 23 de mayo, 2018.

Aceptado: 20 de abril, 20019.

\section{Resumen}

El presente estudio tuvo por objetivo analizar los significados psicológicos que poseen profesores en ejercicio de Chile y que actualmente participan de un proceso de formación continua. La muestra, intencionada no probabilística, estuvo compuesta por 46 docentes, 23 de ellos de enseñanza básica, estudiantes de un postítulo en Ciencias Naturales, y el resto de enseñanza media, con especialidades en Física, Química y Biología, y que en la actualidad reciben capacitación en indagación científica escolar.

La metodología de trabajo utilizada en esta investigación, fue la técnica de "Redes Semánticas Naturales" (Valdez, 2005), la cual busca identificar la influencia de la cultura en la construcción psicológica de identidades.

Se concluye que los profesores en estudio muestran creencias tradicionales y positivistas de la ciencia, y conciben una ciencia de enseñanza algorítmica y protocolar, basada en la transmisión de conceptos y en la construcción de un pensamiento científico definitivo, estático, verdadero y absoluto.

\section{Abstract}

Analysis of the psychological meaning of the concept science in primary and secondary school teachers: an approach from an experience of continuous training

The aim of the study was to analyze the psychological meanings of current teachers participating in a process of continuous training. The intentional, non-probabilistic sample was composed of graduate students of the Natural Sciences program (Valdez, 2005), 46 teachers -23 of them elementary education teachers and 23 secondary teachers specializing in Physics, Chemistry and Biology-, currently being trained in scholastic scientific research.

The methodology used in this research was the technique of Natural Semantic Networks (Valdez, 1998), a tool that seeks to identify the influence of culture in the psychological construction of identities.

The study concludes that the teachers in the study displayed traditional, positivist beliefs on science. They conceive a science of algorithmic and formal teaching, based on the transmission of concepts and the construction of definitive, static, true, and absolute scientific thinking.

1 El Dr. Cristian Aguilar es graduado como Profesor de Educación General Básica, como Magister en Educación de las Ciencias y como Doctor en Didáctica de las Ciencias Experimentales, en la Universidad Complutense de Madrid. En la actualidad, trabaja en el Departamento de Formación Inicial Escolar de la Facultad de Ciencias de la Educación, de la Universidad Católica del Maule-Chile. Contacto: caguilar@ucm.cl.

\section{PALABRAS CLAVE:}

ciencia, memoria colectiva, docentes, identidad, mente, perfeccionamiento.

KEY WORDS:

science, collective memory, teachers, identity, mind, improvement. 


\section{INTRODUCCIÓN}

El progreso científico de los últimos años ha permitido transformar el modo en que se ve y cada uno se sitúa en el mundo. La Humanidad ha transitado desde una sociedad industrial a otra del conocimiento: vertiginosa, cambiante e incierta. Las fuentes de datos son cada día más diversas y los flujos de información atraviesan el globo en cuestión de segundos. Sin embargo, el trabajo científico escolar continúa desarrollándose desde un modelo pedagógico tradicional (Cofré, Galaz, García, Honores, Moreno, Andrade y Vergara, 2009) y en un escenario caracterizado por la transmisión de datos y el aprendizaje de conceptos estériles y acabados, relegando, a un segundo o tercer plano, el desarrollo de habilidades, destrezas y actitudes científicas (Pujol, 2007; Izquierdo, 2007; Sanmartí, 2009).

El proceso de enseñanza actual, referido a educación científica escolar, está determinado por una didáctica que no estimula el desarrollo global de los educandos, este es más bien un acto pedagógico impuesto desde afuera, donde la separación es tan grande que los contenidos y los métodos de enseñanza utilizados son ajenos a las reales necesidades educativas que poseen los jóvenes estudiantes (Dewey, 2004). Quizás, las ideas más plausibles de esta problemática, se fundamenten en principios didácticos orientados hacia una educación científica con foco en el paradigma tradicional (Cofré et al., 2009) en las que el alumno actúa como receptor pasivo de unos datos atomizados, distantes y llenos de sin sentido (Porlán, 1989; Smith y Neale, 1991; Ballenilla, 1992; Pomeroy, 1993; Powell, 1994).

Respecto de la transformación de la enseñanza de las ciencias en los territorios escolares, existe evidencia que el trabajo de los maestros es la vía más efectiva para desarrollar una real alfabetización científica en la población escolar (Zahur, Barton y Upadhyay, 2002; Hattie, 2003; Haberman, 2006; González, Martínez y Martínez, 2009). Sin embargo, a pesar de los cambios producidos en la filosofía de la ciencia y en su didáctica, la enseñanza llevada a cabo por los maestros, sobre todo en niveles primarios y secundarios, continúa reflejando, a menudo, un trabajo áulico, cargado de significados psicológicos de naturaleza positivista, inminentemente transmisivos y recurrentemente conceptuales (Hodson, 1988; Gar-
cía-Carmona, Vázquez y Manassero, 2011; Pujalte, Adúriz-Bravo, y Porro, 2015).

En este contexto argumentativo, se debe señalar que el pensamiento del profesor, $y$, en concreto, sus teorías, creencias y significados, en nuestro caso, sobre la enseñanza y el aprendizaje de las ciencias, guiarán su labor docente y condicionarán, de forma determinante, los acontecimientos pedagógicos que ocurrirán en el aula (García-Carmona, Vázquez y Manassero, 2011). Ello admite, por lo tanto, comprender que el paso de un saber sabio a un saber enseñado (Chevallard, 2000) no solo requiere de una reestructuración del armazón didáctico en su totalidad, sino también de una reestructuración de los significados epistemológicos que poseen los maestros.

Por lo anteriormente expuesto, se derivan entonces las pregunta de esta investigación: ¿Qué significados psicológicos poseen los docentes que hacen ciencia sobre la ciencia?, ¿qué imágenes subyacen en ellos en relación al pensar, al sentir y al actuar científico después de años trabajando en el sistema educativo?, ¿desde qué significados construyen, deconstruyen y fundamentan el trabajo científico?, ¿hay imaginarios colectivos que se levantan socialmente?

No está de más anotar que educar a las actuales y futuras generaciones desde nuevos planteamientos epistemológicos es una acción urgente y necesaria. Entregar nuevas herramientas culturales al estudiantado, a objeto de que puedan construir su propio discurso social, cultural y natural, y así aportar en la construcción de un mundo más humano, justo y sostenible es una acción apremiante y a la cual los profesores que hacen ciencias no deben renunciar.

\section{ENSEÑANZA Y APRENDIZAJE DE LAS CIENCIAS EN EL SISTEMA ESCOLAR. ¿QUÉ CIENCIA APRENDEN LOS ESTUDIANTES?}

La necesidad de mejorar la enseñanza y el aprendizaje de las Ciencias Naturales en la educación obligatoria es un acto urgente. Así lo confirman las investigaciones del último tiempo (Zahur Zahur, Barton y Upadhyay, 2002; Hattie, 2003; Haberman, 2006; Pujol, 2007; Izquierdo, 2007; Sanmartí, 2009; Cofré et al., 2010; Ravanal y Quintanilla, 2012; JiménezTenorio y Oliva, 2016 y Acevedo y García-Carmona, 2016). Los resultados de pruebas estandarizadas llevadas a cabo a nivel nacional e internacional en 
esta área del conocimiento, revelan que, en general, los estudiantes solo alcanzan niveles de aprendizaje que van de lo muy simple y elemental a aprendizajes intermedios (TIMSS, 2011; TERCE, 2013; SIMCE, 2014; PISA, 2014). Si bien los estudiantes, poseen algunos conocimientos de naturaleza científica, solo son capaces de aplicarlos a situaciones en contextos que les resultan muy familiares y avalados por razonamientos directos e interpretaciones muy literales (Cariola, Cares, Lagos, Covacevich y Gubler, 2009).

Por su parte, tampoco son muy alentadores los resultados en relación al desarrollo de Habilidades de Pensamiento Científico (HPC). Las estrategias que utilizan frecuentemente los estudiantes, tanto en el plano educativo, como en la vida cotidiana, van de un manejo básico de indagaciones a procedimientos muy simples en la resolución de problemas (SIMCE, 2014; PISA, 2014; TIMSS, 2011; TERCE, 2013). Del mismo modo, la necesidad de potenciar desde esta área del conocimiento, el desarrollo de nuevos valores y actitudes ciudadanas ha sido otro de los grandes temas pendientes (PISA, 2014; TIMSS, 2011; TERCE, 2013).

La educación científica actual se fundamenta desde un escenario didáctico desde el cual muy raramente se intenta desarrollar el espíritu crítico y reflexivo en los estudiantes, es más bien una enseñanza en donde se les obliga a repetir y a creer (Giordan, 1993; Giordan y De Vecchi, 1997; Bachelard, 2007). En consecuencia, la enseñanza científica en nuestras aulas está determinada por un enfoque didáctico centrado en el aprendizaje de certezas y fundamentado desde un modelo pedagógico que privilegia un saber adiestrado, un pensar dirigido y un conocimiento acabado e inconexo.

Ahora bien, la necesidad de mejorar y potenciar la educación científica en la escuela está reconocida ampliamente (Giordan, 1993; Pujol, 2007; Izquierdo, 2007; Sanmartí, 2009; Cofré et al., Ravanal y Quintanilla, 2012; Jiménez-Tenorio y Oliva, 2016 y Acevedo y García, 2016). Hoy nadie pone en duda su importancia en la formación de los escolares, y las consecuencias de sus aportes en la construcción de una sociedad más sostenible. En la actualidad, es primordial favorecer una real alfabetización científica en el alumnado. Los estudiantes deben tener la capacidad de utilizar el conocimiento para identificar preguntas, adquirir nuevas ideas, explicar fenómenos científicos, pensar teóricamente y sacar conclusiones basadas en evidencias respecto de temas relativos a la ciencia (Mineduc Chile, SIMCE, Unidad de Currículum y evaluación, PISA 2009).

Adquirir estos saberes, no solo se trata de registrar una serie de informaciones datos, fechas, años, hechos. Es, sobre todo, configurar un proceso humano de aprendizaje que es integrador, cambiante, activo y relacional (Ausubel, 2009).

Por tales motivos, puede decirse que la meta de la educación científica escolar no es aprehender un cuerpo de conceptos, hechos y teorías vagas e inconexas; es más bien una progresión hacia ideas clave que permitan entender eventos y fenómenos de relevancia en la vida del sujeto. Los estudiantes deben ser capaces de comprender las grandes ideas sobre objetos, fenómenos, materiales y sobre las complejas relaciones que caracterizan a la naturaleza. En definitiva, se trata, de que los educandos aprendan a construir su propio discurso científico y a ser capaces de hablar de los fenómenos de su mundo (Sanmartí, 2009).

\section{ENSEÑANZA DE LAS CIENCIAS Y FORMACIÓN DEL PROFESORADO ¿QUÉ CIENCIA PIENSAN Y TRANSMITEN LOS PROFESORES DE CIENCIAS?}

Vygotski (2009), reconoce la existencia de un mundo interno que los sujetos construimos, a partir de un proceso de internalización, de un mundo externo. Esta construcción, se halla en las distintas conexiones que el hombre genera a partir de los signos, los cuales cumplen un papel primordial en la comprensión y construcción de los significados. Vygotski (2009) indica que, si los signos se encuentran en la cultura, los significados son parte consustancial de esa cultura. En consecuencia, para el autor, los significados se construyen mediante la internalización de los procesos psicológicos superiores, cuyo valor radica en la capacidad de crear estímulos artificiales que pasan finalmente a ser causas inmediatas de nuestras conductas (Vygotski, 2009). Es en esta dialéctica que los individuos organizan las ideas, interpretan el mundo y le otorgan sentido a lo que hacen.

En efecto, atribuir valor a un concepto, a un objeto, a un signo implica ponerlo en estrecha interacción 
con otros elementos y con el medio adyacente (Vera, Pimentel y Batista De Albuquerque, 2005).

La escuela es uno de esos espacios sociales en donde se puede transmitir una herencia cultural de tipos específicos de conocimientos, en nuestro caso el científico. Para un número considerable de estudiantes, este conocimiento constituye un saber complejo y dogmático, expresado en un lenguaje difícil y relegado solo a algunos pocos (Pujol, 2007).

Desde los primeros años de formación escolar los estudiantes aprenden a aislar los ámbitos del saber. Son formados para separar unos aprendizajes de otros y así, pierden la aptitud de conectar, y, por ende, la capacidad de pensar los problemas desde una realidad compleja, dinámica y multicausal (Morín y Hulot, 2008).

Además, algunas corrientes actuales, sobre enseñanza y aprendizaje de las Ciencias Naturales, se caracterizan por ser reduccionistas (Morín, 2007), ya sea en sus bases epistemológicas, como en los fundamentos didácticos sobre los cuales se sostienen. Términos como "producto formal y terminado", "posición racionalista" o "transmisión de conocimiento enciclopédico" caracterizan actualmente la llamada epistemología conservadora de la ciencia (Porlán y Martín del Pozo, 2004).

En un modelo pedagógico en donde la enseñanza se concibe como el acto de transmitir datos, los estudiantes aprenden a repetir datos. En estos entornos didácticos se considera que el deber de los educandos es hacer y aprender como (Dewey, 2004). Esto significa ser depósitos (Freire, 2008), de lo que ya está incorporado en los libros de texto, o bien, en la mente de sus profesores. El nuevo planteamiento de la enseñanza científica escolar debe aspirar a promover el desarrollo global de las capacidades del sujeto. Sin embargo, existe una ausencia de reflexión sobre cuáles son sus metas y finalidades en la formación de los estudiantes (Pujol, 2007). En efecto, cabe preguntarse ¿cuál es el valor de la educación científica en el sistema escolar?, ¿cómo transitamos desde una educación científica inminentemente conceptual a una educación que propicia el desarrollo de habilidades, actitudes y sistema de valores en la escuela?, ¿qué ciencia piensan y transmiten los docentes que hacen ciencias?, ¿para qué sujetos?, ¿para qué so- ciedad?, ¿están preparados los docentes para asumir estos retos?

Los magros resultados sobre rendimientos escolares asociados a la enseñanza y el aprendizaje de las Ciencias Naturales (TIMSS, 2011; TERCE, 2013; SIMCE, 2014; PISA, 2014), han dirigido la atención hacia la formación inicial y continua de profesores (Ministerio de Educación de Chile, 2005). La investigación en esta línea nos indica que, por lo general, los docentes tienen una concepción de ciencia basada en la búsqueda de certezas, en la trasmisión de un conocimiento indiscutible y en donde la función de la observación y la experimentación se limitan a comprobar una verdad explicada (Sanmartí, 2009).

García-Carmona, Vázquez y Manassero (2011), por su parte, afirman que los maestros atribuyen al conocimiento científico un valor protocolar; definitivo, estático, verdadero y absoluto. Ello supone que en sus prácticas pedagógicas los docentes siguen utilizando una metodología de enseñanza que se basa simplemente en la transmisión de conceptos y en la memorización de los contenidos (Olivia y Acevedo, 2005). Al respecto, Quintanilla (2003) señala que "la concepción de los docentes sobre ésta [sic] área continúa siendo ingenua, pues la consideran como un instrumento de los procesos de enseñanza más tradicionales y dogmáticos" (p. 2). En definitiva, se arguye que los profesores muestran creencias tradicionales, positivistas e idealistas respecto de cómo enseñar ciencias, y asumen una comprensión de ella desde sus trincheras de especialización, como puede ser la biología, la física y la química, etc. (García-Carmona, Vázquez y Manassero, 2011). Porlán y Martín del Pozo (2004) Ilamarían a este tipo de ciencia una "ciencia del conocimiento", generada por procesos técnicos que se circunscriben a una metodología basada en actividades en las cuales los estudiantes deben aplicar rigurosamente los pasos del método científico.

Lo anterior cobra relevancia y sentido, pues la comprensión y los significados individuales y culturales que los profesores tienen sobre la naturaleza de la ciencia y su didáctica es un factor relevante respecto de lo que acontecerá en el proceso de enseñanza de la misma (García-Carmona, Vázquez y Manassero, 2011). Estas ideas son las que los docentes transmiten cuando enseñan ciencias (Fernández, Gil Pérez, 
Carrascosa, Cachapuz y Praia, 2002; Pujalte, AdúrizBravo y Porro 2015). Y estas, a su vez irisarán la ciencia a través de la cual los sujetos leen e interpretan la realidad circundante.

\section{MARCO METODOLÓGICO}

\section{Participantes}

Se consideró una muestra intencionada de profesores que en la actualidad participan en programas de formación continua desarrollados en dos universidades chilenas presentes en la Región del Maule.

La muestra total estuvo compuesta por 46 docentes: 23 de enseñanza básica, licenciados en educación y estudiantes de un postítulo en Ciencias Naturales, y el resto de enseñanza media, todos licenciados y con especialidades en Física, Química y Biología, estos últimos, participantes de un programa formativo relacionado con indagación científica escolar.

Del total de docentes, 37 eran mujeres $(80.4 \%)$ y 9 (19.6\%) varones, con una edad promedio que bordeaba los 38 años.

Resulta importante de señalar que este grupo trabaja tanto en contextos educativos rurales como urbanos, en los niveles primarios y secundarios, en establecimientos mayoritariamente de dependencia pública y privada subvencionada, y en las provincias de Talca, Linares y Curicó principalmente.

\section{Diseño}

El diseño del estudio fue descriptivo comparativo y utilizó una metodología mixta, mediante la técnica de Redes Semánticas Naturales (RSN) (Valdez, 2005).

\section{Instrumento}

El instrumento utilizado para recabar la información consideró el uso de las RSN. Esta herramienta investigativa intenta explicar cómo ocurre la construcción de significados en los individuos (Valdez, 2005).

Para las RSN, la información almacenada en la memoria está organizada en nodos conceptuales, que en conjunto crean un código personal de reacción cognoscitiva, reflejando de esta manera la imagen simbólica que los sujetos construimos respecto del mundo que transitamos. Las RSN ofrecen una metodología práctica de trabajo con el fin de poder vislumbrar cómo los sujetos organizan el conocimiento en su estructura cognitiva (Ausubel, 2009) y, cómo a partir de esta internalización (Vygotski, 2009) el significado es construido desde un escenario cargado de entidades socio individuales.

A grandes rasgos, la técnica permite que los participantes puedan definir, individualmente y de manera rápida y espontánea, un concepto dado. Para ello, deben utilizar un mínimo de cinco palabras que posteriormente jerarquizarán de acuerdo con la importancia, cercanía o ligazón que ellos consideren tiene su red conceptual en relación con el estímulo propuesto. Finalmente, asignan el número 1 a la palabra más cercana, el 2 a la que sigue y así sucesivamente hasta terminar con el número 5 que sería el concepto más alejado del troncal fijado.

Para el caso de esta investigación, los docentes debieron definir libremente y con un tiempo máximo de diez minutos, el concepto nodal "Ciencia".

Durante la primera sesión de su proceso formativo cada uno de los profesores recibió un folio en donde aparecía escrito el concepto indicado precedentemente y bajo este, cinco rayas dispuestas en columna (aquí indicaban la red conceptual) cada una de las cuales se acompañaba a su derecha de otra más pequeña (donde señalaban el número de la jerarquía).

Una vez finalizado el proceso anterior y con la red definida y jerarquizada, cada participante debía manifestar (en no más de cinco líneas) algunas ideas que explicaran las razones de la red construida y los juicios que le llevaron a jerarquizar las palabras de ese modo.

Esta última información resulta valiosa pues complementa acciones tendientes a la triangulación de los datos y otorga mayor solidez y coherencia a las conclusiones.

Finalmente, y para una mayor claridad en la presentación de los datos, se utilizará la propuesta de trabajo indicada por Lagunes (1993) y Valdez (2005), quienes describen los siguientes indicadores básicos: conjunto SAM, grupo de las diez palabras definidas por los docentes que obtienen los mayores valores M. Valor J o tamaño de la red (TR), valor que se obtiene sumando el número de palabras que fueron definidas, por cada uno de los grupos, respecto del concepto dado, peso semántico (valor M. Este corresponde al valor de la frecuencia, asignado por los 
docentes a cada concepto, por el valor semántico dado por los autores de la técnica (valor $5=1$; valor $4=2$; valor $3=3$; valor $2=4$ y valor $1=5)$ ) y finalmente, la diferencia semántica cuantitativa (DSC) y el indicador (en porcentaje), de la distancia semántica que hay entre las diferentes palabras que conforman la red (este valor se obtiene mediante una regla de tres simple, tomando como inicio la palabra con el valor M mayor, la cual representa el 100\%).

En segundo término, se presenta un gráfico radial que detalla la configuración de la red conceptual definida y cuyo valor, radica en revelar las distancias semánticas en las agrupaciones de palabras generadas por los grupos en estudio.

\section{RESULTADOS}

En términos de la muestra total (véase la Tabla 1), se puede afirmar que se aprecia que el núcleo de la red quedó representado por el concepto "Conocimientos".

Seguidamente, y con un peso semántico del (84\%) aparece la palabra "Observación", y muy ligada a esta en términos porcentuales le sigue el concepto "Investigación" (82\%). Luego se presenta la palabra "Experimentos", con un peso semántico del $64 \%$ y después los conceptos "Estudio" y "Naturaleza" con valores de $45 \%$ y $44 \%$ respectivamente. Seguidamente el concepto de "Descubrir" (29\%) y después, los términos con valores del 23\%: "Asignaturas" y "Vida". Finalmente aparece la palabra "Hipótesis" con un valor porcentual del $21 \%$.

Es importante recordar que el tamaño de la red semántica (valor J) estuvo compuesta por un total de 54 definidoras.

Tabla 1. Conjunto SAM concepto Ciencia. Muestra total

\begin{tabular}{lll}
\hline \multicolumn{1}{c}{ Palabras definidoras } & M & DSC \\
\hline Conocimientos & 66 & $100 \%$ \\
\hline Observación & 56 & $84 \%$ \\
\hline Investigación & 54 & $82 \%$ \\
\hline Experimentos & 42 & $64 \%$ \\
\hline Estudio & 30 & $45 \%$ \\
\hline Naturaleza & 29 & $44 \%$ \\
\hline Descubrir & 19 & $29 \%$ \\
\hline Asignaturas & 15 & $23 \%$ \\
\hline Vida & 15 & $23 \%$ \\
\hline Hipótesis & 14 & $21 \%$ \\
\hline
\end{tabular}

Valor J o TR $=54$

Fuente: elaboración propia

En cuanto a los significados del concepto "Ciencia" (véase la Tabla 2) para los profesores de enseñanza media en las aéreas de Química, Física y Biología, el núcleo de la red quedó dado por el concepto troncal "Observación". Les siguen los términos "Conocimientos" (86\%) e "Investigación" (80\%) ambos con pesos semánticos elevados.

También figuran con pesos semánticos interesantes las palabras "Naturaleza" (66\%) y "Experimentos" (58\%). Luego, están los conceptos de "Estudio" y
"Metodología", con valores porcentuales del 36\% y $30 \%$ respectivamente, y seguidamente "Aprendizaje" y "Teoría" con valores cercanos al 25\%. Finalmente, con un peso semántico del 19\% figura el concepto "Búsqueda".

El tamaño de la red conceptual (valor J) para este colectivo de docentes, estuvo compuesta por un total de 42 definidoras. 


\section{Gráfico radial 1. Valores $\mathrm{M}$ muestra total}

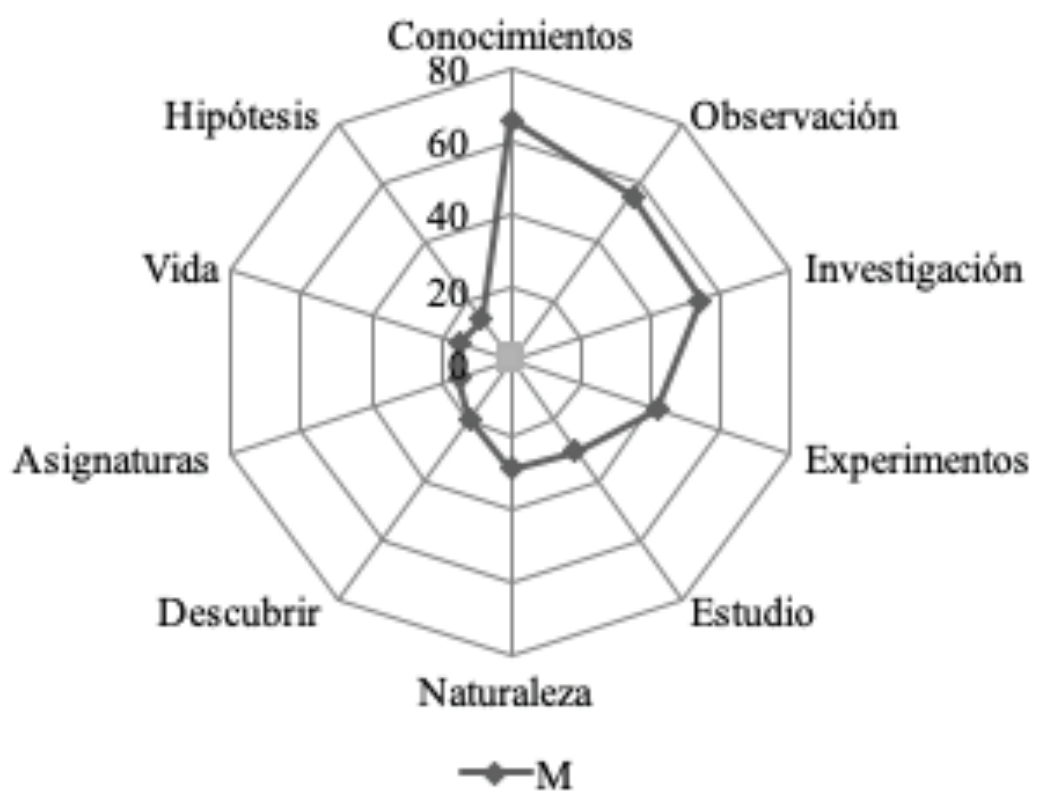

Fuente: elaboración propia

Tabla 2. Conjunto SAM concepto Ciencia. Profesores de Química, Física y Biología

\begin{tabular}{lcc}
\hline \multicolumn{1}{c}{ Palabras definidoras } & M & DSC \\
\hline Observación & 36 & $100 \%$ \\
\hline Conocimientos & 31 & $86 \%$ \\
\hline Investigación & 29 & $80 \%$ \\
\hline Naturaleza & 24 & $66 \%$ \\
\hline Experimentos & 21 & $58 \%$ \\
\hline Estudio & 13 & $36 \%$ \\
\hline Metodología & 11 & $30 \%$ \\
\hline Aprendizaje & 9 & $25 \%$ \\
\hline Teoría & 9 & $25 \%$ \\
\hline Búsqueda & 7 & $19 \%$
\end{tabular}

Fuente: elaboración propia

Por su parte, para los profesores de enseñanza básica (postítulo en Ciencias Naturales), el núcleo del significado psicológico del concepto ciencia (véase la Tabla 3) quedó constituido nodalmente por la palabra "Conocimientos" (100\%). Posteriormente está la palabra "Investigación" con valor porcentual del (74\%) y luego la red de conceptos "Observación" (59\%), "Estudio" y "Experimentos" con valor del 50\%. También la palabra "Asignaturas" (44\%) está en este gru- po y el término "Descubrir" (35\%). Finalmente, hay un nodo con los conceptos "Hipótesis", "Avances" y "Curiosidad", con valores porcentuales del $29 \%$ y $26 \%$ respectivamente.

En este último caso, el tamaño de la red semántica (valor J) para este grupo de profesionales estuvo compuesta por un total de 39 definidoras. 


\section{Gráfico radial 2. Valores M. Profesores de Química, Física y Biología}

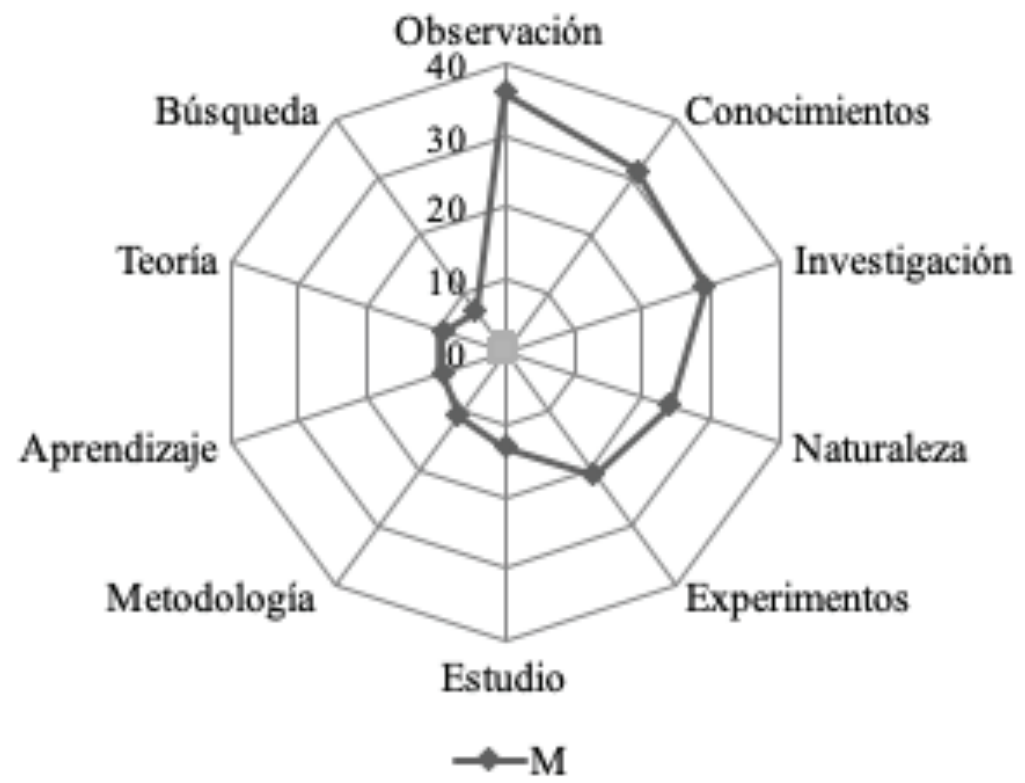

Fuente: elaboración propia

Tabla 3. Conjunto SAM concepto ciencia. Profesores de Educación General Básica con postítulo en Ciencias Naturales

\begin{tabular}{lcc}
\hline \multicolumn{1}{c}{ Palabras definidoras } & M & DSC \\
\hline Conocimientos & 34 & $100 \%$ \\
\hline Investigación & 25 & $74 \%$ \\
\hline Observación & 20 & $59 \%$ \\
\hline Estudio & 17 & $50 \%$ \\
\hline Experimentos & 17 & $50 \%$ \\
\hline Asignaturas & 15 & $44 \%$ \\
\hline Descubrir & 12 & $35 \%$ \\
\hline Hipótesis & 10 & $29 \%$ \\
\hline Avances & 9 & $26 \%$ \\
\hline Curiosidad & 9 & $26 \%$ \\
\hline
\end{tabular}

Valor J o TR $=39$

Fuente: elaboración propia 


\section{Gráfico radial 3. Valores M. Profesores de}

\section{Educación General Básica con postítulo en Ciencias Naturales}

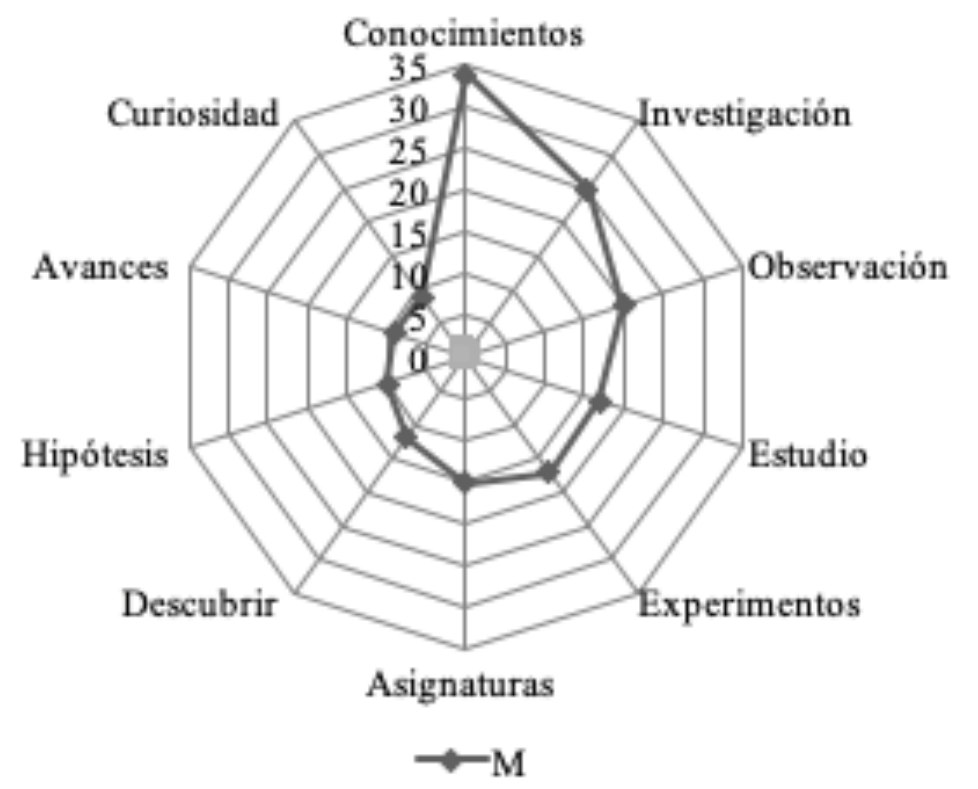

Fuente: elaboración propia

\section{DISCUSIÓN}

La investigación se enfocó en analizar los significados psicológicos, sobre el concepto ciencia, que posee un grupo de docentes en ejercicio (enseñanza básica y media) y que formaban parte de una experiencia de perfeccionamiento continuo.

La mayor riqueza semántica (aunque muy estrecha) respecto del Valor J fue para el grupo de profesores de enseñanza media (TR, 42), mientras que la red conceptual con menos definidoras fue para el grupo de profesores de enseñanza básica (TR, 39). Por su parte, las distancias semánticas (DSC) más homogéneas fueron para el conjunto de docentes vinculados a las pedagogías de enseñanza media a diferencia de los maestros de primaria en donde se muestran DSC más dispersas.

Para el común de los maestros participantes en el estudio, independiente de su profesión, especialidad y nivel educativo en el que laboran, la ciencia es significada hacia el concepto "Conocimientos".

De acuerdo con la literatura, una enseñanza materializada bajo este paradigma propende a la generación de un entorno didáctico gravitado por signifi- cados de naturaleza transmisiva y una epistemología de tipo positivista (Hodson, 1988; García-Carmona, Vázquez y Manassero, 2011; Pujalte et al., 2015). En efecto, enseñar ciencias, podría simbolizar para este colectivo de profesores, instruir datos, repetir fechas, anécdotas y aprender conceptos. Porlán y Martín del Pozo (2004) Ilamarían a este tipo de ciencia", ciencia del saber y del conocer", ciencia generada a partir de procesos pedagógicos instrumentalistas e inminentemente operativos.

Otro nodo conceptual interesante, surgido en las redes semánticas, nos releva hacia una familia de palabras como la "Observación", la "Investigación" y la "Experimentación". Habilidades troncales e indispensables en el desarrollo de la alfabetización científica-escolar, sobre todo en las primeras edades. Sin embargo, los datos debemos valorarlos en su justa medida. Investigaciones en la línea declarada, nos muestran que la observación y la experimentación científica desarrollada en la escuela, en la mayoría de las veces, se limita a comprobar una verdad explicada (Sanmartí, 2009), verdad por lo común, dada por una figura de autoridad el profesor y otra dada en términos de contenido, significada principalmente desde lo declarado en los libros de clases. 
El carácter de ciencia Asignaturista, que nos muestra la red semántica total, viene a reforzar lo que ya habíamos indicado. Enseñar ciencias es quizás transmitir datos y aprender conceptos. Estos hallazgos coinciden con trabajos Ilevados a cabo por GarcíaCarmona, Vázquez y Manassero (2011), quienes arguyen que los profesores muestran creencias tradicionales e idealistas respecto de cómo enseñar ciencias y asumen una comprensión de la misma principalmente desde sus áreas de especialización, con la cual empobrecen, quizás, los espacios de aprendizaje al atomizar las áreas del conocimiento cuyo valor radica en estar al servicio de los aprendizajes desde una mirada conectiva e interdisciplinar.

Otro núcleo semántico que Ilama la atención, es que los profesores del estudio significan la idea de ciencia desde elementos figurativos ligados a la "Naturaleza". Hablar de ciencia es traer la naturaleza a la mano. Esto está muy bien, pues la ciencia debe propender a capacitar a los jóvenes estudiantes para que puedan, por una parte, decidir informadamente y, por otro lado, comprender el mundo que transitan, decodificando los lenguajes por medio de los cuales la naturaleza nos habla constantemente. Sin embargo, no deben dejarse de lado otros elementos imbricados a la ciencia y al quehacer científico, como podrían ser algunos elementos epistemológicos, sociales, culturales e históricos.

Conceptos como "Búsqueda", "Curiosidad" y "Descubrir", en ciencias se refieren más al hacer de los estudiantes que al de los maestros. Además, aparecen relegados a un segundo o tercer plano, y así queda de manifiesto en las redes surgidas; las DSC, de estos términos son significativamente inferiores respecto de conceptos como "Conocimientos" e "Investigación".

Finalmente se debe destacar que los conceptos "Descubrir" y "Curiosidad" solo surgen en la red de significados generada por los profesores de enseñanza básica. Quizás para este colectivo de docentes, estas habilidades científicas, tan propias de los estudiantes de los primeros años formativos, son inherentes a su naturaleza comportamental $y$, por tanto, significan no escindirlas de los procesos didácticos ligados a la enseñanza científica escolar en la infancia.

\section{CONCLUSIONES}

De acuerdo con los resultados obtenidos, se visualiza, por una parte, que si bien hay elementos comunes de significado psicológico para la idea de ciencia (en cada grupo participante), también coexisten en ellos una serie de significados alternativos y diferenciadores.

Las redes conceptuales construidas por los profesores varían en función de la carrera cursada, su formación pedagógica universitaria de base, la naturaleza que define a su especialidad y el nivel educativo que atienden.

Para el profesorado de enseñanza media hacer ciencia en la escuela significa mayor manejo y dominio de ciertos saberes disciplinares y normativos a diferencia del profesorado con foco en la enseñanza básica, en donde se valoran más aspectos vinculados hacia lo pedagógico y lo didáctico, sobre todo acentuado para el primer ciclo básico.

El estudio reveló que en general, los docentes significan una ciencia inminentemente ligada al conocimiento, una ciencia algorítmica, fundamentada desde un único método. Pensar y hacer ciencias, es seguir y aplicar rigurosamente los pasos del método científico. En efecto, enseñar ciencias quedaría relegado al acto didáctico de transmitir datos ya elaborados; hacer ciencias es aprender conceptos, fórmulas y teorías.

Para los profesores del estudio, independiente de su especialización, los experimentos y el laboratorio escolar jugarían un rol primario y protagónico en el trabajo científico escolar y estarían relegándose a planos secundarios el uso de los espacios urbanos y naturales, como escenarios pedagógicos ligados a la enseñanza científica escolar.

Por tanto, es apremiante que los maestros continúen formándose a lo largo de su trayectoria profesional. Ser parte de ciclos de perfeccionamiento prolongados favorece la aparición de comunidades profesionales de aprendizaje, de trabajo en redes y de intercambio constante de testimonios y experiencias pedagógicas. Transitar por estas vías además, implica encaminarse hacia la transformación de nuestros propios significados y prácticas pedagógicas. Adquirir estos saberes, no sólo es registrar una serie de datos, hechos y formulas inconexas, es sobre todo configurar un 
proceso de aprendizaje que es integrador, activo y relacional. El valor de la ciencia es ese: una actividad humana bañada por elementos sociales y culturales en constante evolución y dinamismo.

Finalmente, se destaca que el sistema escolar de Chile necesita cada vez más docentes con altas capacidades disciplinares y pedagógicas, a objeto de formar un colectivo de estudiantes que logren enfrentar una sociedad compleja y en constante evolución. Saber qué significados psicológicos representa el pensar y el hacer en ciencias para un profesor en ejercicio es, también, apremiante.

A partir de los resultados obtenidos, surge la necesidad como desde la academia y la universidad nos hacemos cargo de lo hallado y como al mismo tiempo somos capaces de emprender programas de formación continua para el profesorado que sean útiles, pertinentes y contextuales.

\section{REFERENCIAS BIBLIOGRÁFICAS}

Acevedo J. A., García-Carmona A. (2016). Algo antiguo, algo nuevo, algo prestado. Tendencias sobre la naturaleza de la ciencia en la educación científica. Revista Eureka sobre Enseñanza y Divulgación de las Ciencias, 13(1), 3-19.

Ausubel, D. (2009). Adquisición y retención del conocimiento. Una perspectiva cognitiva. Barcelona: Paidós.

Bachelard, G. (2007). La formación del espíritu científico. Contribución a un psicoanálisis del conocimiento objetivo. México: Siglo XXI.

Ballenilla, F. (1992). El cambio de modelo didáctico, un proceso complejo. Investigación en la Escuela, (18), 43-68.

Cariola, L., Cares, G., Lagos, E., Covacevich, C. y Gubler, J. (2009). ¿Qué nos dice PISA sobre la educación de los jóvenes en Chile? Nuevos análisis y perspectivas sobre los resultados en PISA 2006. Santiago de Chile. Ministerio de Educación, Unidad de Curriculum y Evaluación, SIMCE.

Chambers, D. (1983). Stereotypic images of the scientist: The Draw-A-Scientist Test. Science Education, 67(2), 255-265.
Chevallard, Y. (2000). La transposición didáctica. Del saber sabio al saber enseñado. Buenos Aires: Aique.

Cofré, H., Camacho, J., Galaz, A., Jiménez, J., Santibáñez, D. y Vergara, C. (2010). La educación científica en Chile. Debilidades de la enseñanza y futuros desafíos de la educación de profesores de ciencia. Estudios Pedagógicos. 36(2), 279-293.

Cofré, H., Galaz, C., García, C., Honores, M., Moreno, L., Andrade, L. y Vergara, C. (2009). Frecuencia y tipo de actividades de laboratorio que realizan profesores/as primarios en el área de las ciencias, en Santiago de Chile. Enseñanza de las Ciencias, Número Extra VIII Congreso Internacional sobre Investigación en Didáctica de las Ciencias, Barcelona, pp. 3432-3435. Disponible en: https://ddd.uab. cat/pub/edlc/edlc_a2009nEXTRA/edlc_a2009nExtrap3420.pdf.

Dewey, J. (2004). Experiencia y educación. Madrid: Biblioteca Nueva.

Fernández, I., Gil Pérez, D., Carrascosa, J., Cachapuz, A. y Praia, J. (2002) Visiones deformadas de la ciencia transmitidas por la enseñanza. Enseñanza de las ciencias, 20(3), 477- 488.

Fernández, I; Gil Pérez, D; Valdés, P; Vilches, A. (2005). ¿Qué visiones de la ciencia y la actividad científica tenemos y transmitimos? En: Gil Pérez, D y otros. (Comp.). ¿Cómo promover el interés por la cultura científica? Una propuesta didáctica fundamentada para la educación científica de jóvenes de 15 a 18 años (pp.29-62). UNESCO.

Freire, P. (2008). Pedagogía del oprimido. Madrid: Siglo XXI.

García-Carmona, A., Vázquez, A. y Manassero, M.A. (2011). Estado actual y perspectivas de la enseñanza de la naturaleza de la ciencia: una revisión de las creencias y obstáculos del profesorado. Enseñanza de las Ciencias, 28(3), 403- 412.

Giordan, A. (1993). La enseñanza de las ciencias. Madrid: Siglo XXI.

Giordan, A. De Vecchi, G. (1997). Los orígenes del saber. De las concepciones personales a los conceptos científicos. Sevilla: Díada.

González, C., Martínez, M. T. y Martínez, C. (2009) La Educación Científica como apoyo a 
la movilidad social: desafíos en torno al rol del profesor secundario en la implementación de la indagación científica como enfoque pedagógico. Estudios Pedagógicos, 25, 63-78.

González, C., Lopez, V., Bravo, P. y Castillo, P. (2009). Científicos jóvenes y sonrientes: la imagen de científico de los estudiantes chilenos de $11^{\circ} \mathrm{y}$ $12^{\circ}$ grado en diferentes contextos escolares. Enseñanza de las Ciencias, Número Extra VIII Congreso Internacional sobre Investigación en Didáctica de las Ciencias, Barcelona, pp. 2550-2554. Disponible en http://ensciencias.uab.es/congreso09/numeroextra/art-2550-2554.pdf.

Haberman, M. (2006). The special role of science teaching in schools serving diverse children in urban poverty. En: Flick, L \& N. Lederman (eds.), Scientific inquiry and the nature of science: Implications for teaching, learning, and teacher education (pp. 3753). Netherlands: Springer.

Hattie, J. (2003). Teachers make a difference: What is the research evidence? Paper presented at the Australian Council for Educational Research Annual Conference on Building Teacher Quality, Melbourne. Australia. Descargado desde http://research. acer.edu.au/research_conference_2003/4/.

Hodson, D. (1988). Toward a philosophically more valid science curriculum. Science Education, 72(1), 19-40.

Izquierdo, M. (2007). Enseñar ciencias, una nueva ciencia. Enseñanza de las Ciencias Sociales, 6, 125 $-138$.

Jiménez-Tenorio, N. y Oliva, J. M. (2016). Aproximación al estudio de las estrategias didácticas en ciencias experimentales en formación inicial del profesorado de Educación Secundaria: descripción de una experiencia. Revista Eureka sobre Enseñanza y Divulgación de las Ciencias, 13(1), 121-136.

Lagunes, I. R. (1993). Las redes semánticas naturales, su conceptualización y su utilización en la construcción de instrumentos. Revista de Psicología Social y Personalidad, 11, 81-97.

Ministerio de Educación de Chile. (2011). Agencia de la Calidad de la Educación. Resumen de resultados TIMSS 2013. Santiago de Chile.
Ministerio de Educación de Chile. (2014). Agencia de la Calidad de la Educación. Resumen de resultados PISA 2014. Santiago de Chile.

Ministerio de Educación de Chile. (2014). Agencia de la Calidad de la Educación. Resumen de resultados SIMCE 2014. Santiago de Chile.

Ministerio de Educación de Chile. (2009). Resumen de resultados PISA 2009. Santiago de Chile.

Ministerio de Educación de Chile. (2005). Bases curriculares de la educación parvularia. Unidad de curriculum y evaluación. Santiago de Chile.

Morín, E. (2007). Introducción al pensamiento complejo. Barcelona: Gedisa.

Morín, E. y Hulot, N. (2008). El año I de la era ecológica. Barcelona: Paidós.

Oliva, J. y Acevedo, J. (2005). La enseñanza de las ciencias en primaria y secundaria hoy. Algunas propuestas de futuro. Revista Eureka sobre Enseñanza y Divulgación de las Ciencias, 2(2), 241-250.

Pomeroy, D. (1993). Implications of teachers' beliefs about the nature of science: Comparison of the beliefs of scientist, secondary science teachers, and elementary teachers. Science Education, 77(3), 261-278.

Porlan, R. (1989). Teoría del conocimiento, teoría de la enseñanza y desarrollo profesional. Las concepciones epistemológicas de los profesores. Tesis Doctoral en Didáctica y Organización Educativa. Universidad de Sevilla.

Porlan, R. y Martín del Pozo, R. (2004). The conceptions of in-service and prospective primary school teachers about the teaching and learning of Science. Journal of Science Teacher Education, 15(1), 3962.

Powell, R. (1994). From field science to classroom science: A case study constrained emergence in a second-career science teacher. Journal of Research in Science Teaching, 31(3), 273- 291.

Pujalte, A., Adúriz-Bravo, A. y Porro, S. (2015). Las imágenes de ciencia en profesores y profesoras de Biología: Entre lo que se dice y lo que se hace. Revista Boletín Biológica, 33(9), 5-10.

Pujol, R. (2007). Didáctica de las ciencias en la educación primaria. Madrid: Síntesis. 
Quintanilla, M. (2003). Equidad y calidad de la educación científica en América Latina. Algunas reflexiones para un debate sobre los modelos de formación inicial y continua de los profesores de ciencia. UNESCO. Santiago de Chile. Disponible en: http://www7.uc.cl/sw_educ/educacion/ grecia/plano/html/pdfs/Formacion_continua/ Seminarios_y_congresos/FPR016.pdf.

Ravanal, E. y Quintanilla, M. (2012). Concepciones del profesorado de biología en ejercicio sobre el aprendizaje científico escolar. Enseñanza de las Ciencias, 30(2), 33-54.

Sanmartí, N. (2009). Didáctica de las ciencias en la educación secundaria obligatoria. Madrid: Síntesis.

Smith, D.C. y Neale, D.C. (1991). The construction of subjet-matter knowledge in primary science teaching. Advances in Research on Teaching, 2, 187243.

UNESCO (2013). Tercer estudio Regional Comparativo e Explicativo (TERCE). UNESCO. Santiago de Chile.

Valdez, J. (2005). Las redes semánticas naturales, uso y aplicaciones en psicología social. México: Universidad Autónoma del Estado de México.

Vera J, Pimentel C, y Batista De Albuquerque, F. (2005). Redes semánticas: aspectos teóricos, técnicos, metodológicos y analíticos. Rev Ra Ximhai, (1), 439-51. Disponible en http://www.revistas. unam.mx/index.php/rxm/article/view/6845.

Vygotski, L. (2009). El desarrollo de los procesos psicológicos superiores. Barcelona: Biblioteca de bolsillo.

Zahur, R., Barton, A. C. y Upadhyay, B. R. (2002). Science education for empowerment and social change: a case study of a teacher educator in urban Pakistan. Int. J. Sci. Educ. 24: 899-917. 